\title{
Analysis of the optical field on the human retina from wavefront aberration data
}

\author{
Sergio Barbero and Susana Marcos
}

\begin{abstract}
Wave aberrations in the human eye are usually known with respect to the ideal spherical wavefront in the exit pupil. Using Kirchhoff's diffraction theory, we have derived a diffraction integral to compute the optical field on the retina from the wave aberration data. We have proposed a numerical algorithm based on the StamnesSpjelkavik-Pedersen (SSP) method to solve that integral. We have shown which approximations are admissible to reduce the complexity of the diffraction integral. In addition, we have compared our results with those of the conventional procedure used to compute intensities on the retina. We have found significant differences between our results and the conventional ones.

OCIS codes: $330.7326,330.5370,050.1970$.
\end{abstract}

\section{INTRODUCTION}

In optical eye modeling, specifically in ophthalmic lens design and visual performance simulations, it is often necessary to compute the intensity of light

on the retina. In both applications, light propagation inside the eye is usually modeled using the single-plane transition method . This method is based on two steps. First, wavefront propagation from the object space to a surface of the image space is modeled using geometrical optics computations or experimentally measurements. Second, having computed or measured the wave aberration in the image space, it is necessary to compute the optical field in the retinal surface, solving a diffraction integral.

This method is valid only if the only diffracted rays reaching the image are rays coming from the aperture stop. In the human eye, this occurs when object points are not very far from the optical axis and the surfaces of the ocular components are not very asymmetric.

As an alternative, it is possible to model light propagation through computation of cascade-aperture diffraction integrals . Perez have proposed this type of algorithm (applying the Debye diffraction theory) for the human eye. However, this procedure is computationally demanding and needs a specific model for the internal morphology of the human eye. Besides, in many cases, the human eye can be modeled as an optical system described by a wave aberration measured experimentally in a reference surface. Thus, the single transition method is still very useful.

In the single transition method, it is typically assumed that the Fraunhofer (or sometimes the Fresnel) approximation to diffraction integrals is sufficiently accurate to compute the intensity on the human retina. However, Fresnel and Fraunhofer approximations are both based on the paraxial approximation, whose validity has been recently questioned with respect to the human eye
In the human eye, wave aberrations are usually measured in a double-pass experimental setup; i.e., light is delivered into the retina, and the reflected light from there outward is collected onto a camera. Depending on the specific technique, the wave aberration is measured in the first pass (e.g., in a laser ray-tracing sensor) or in the second pass (e.g., in a Hartmann-Shack sensor ). In the former, the wave aberration is measured directly in the image space with respect to an ideal spherical wavefront, whereas in the latter the wave aberration is measured in the object space with respect to a plane surface. Moreno-Barriuso and Navarro, and more recently Castro , have shown the experimental equivalence of the wave aberration measured in the second pass in a planar surface and the wave aberration measured in the first pass with respect to the ideal spherical wavefront. Therefore, we can consider that we always know the wave aberration with respect to the ideal spherical wavefront in the image space.

The point-spread function (PSF) of the human eye is typically evaluated assuming that the wave aberration has been measured in either case with respect to the exit planar pupil (image space). However, as mentioned above, the PSF should be evaluated by defining the wave aberration with respect to the ideal reference wavefront in the image space. Therefore, a major goal of this study is to derive the diffraction integrals necessary to compute the PSFs (or more generally the optical field on the retina) with respect to the ideal spherical wavefront instead of with respect to the exit planar pupil. Kraus derived Kirchhoff diffraction integrals by performing the integration over a spherical wavefront surface rather than the aperture plane. However, he did not consider the case of nonspherical wave aberrations. We derived the diffraction integrals in the general case of any type of wave aberration.

We performed an analysis for an efficient and accurate computation of that integral and studied which 
approximations-those applied to simplify the exact diffraction integral-can be justified to reduce the computation effort. Obviously, the validity of the approximations depends on the specific situations. We studied two situations: The axial intensity distribution, when the object is located on axis in the near and far regions (depth-of-focus analysis), and the intensity distribution off axis in the paraxial image plane and in an out-of-focus plane.

In addition, we compared the results using the new procedure versus the conventional procedure. In the conventional procedure, the wave aberration is considered with respect to the exit pupil plane and the PSF is evaluated by applying the Fraunhofer approximation to the exact diffraction integral. The Fraunhofer integral is evaluated numerically by applying a fast-Fouriertransform (FFT) algorithm.

\section{DIFFRACTION INTEGRAL FORMULA}

We assumed that the wavefront aberration function (W), defined as the difference between the real wavefront and the ideal spherical wavefront, was known. Thus, in the scalar theory, the optical field at the ideal spherical wavefront $(\Omega)$ was (see Fig. 1) known and represented by $u$. We assumed that the amplitude of the optical field is constant at $\Omega$. An apodization of the amplitude could be considered to account for the Stiles-Crawford effect . Alternatively, Vohnsen proposed in a recent article to include this effect as a retinal waveguiding transformation of the optical field without the need for any type of pupil apodization.

We used Kirchhoff's diffraction theory . Although Kirchhoff's theory is not as rigorous as Sommerfeld's diffraction theory, it is valid for this case because the linear dimensions of the aperture (exit pupil) are large compared to the wavelength and the observation points are not very close to the optical aperture. Moreover, it can be used in a more straightforward manner because the optical field is known in a nonplanar surface

Kirchhoff's theorem, with Kirchhoff's boundary conditions , establishes that the optical field at point $O$ (Fig. 1) is given by the equation

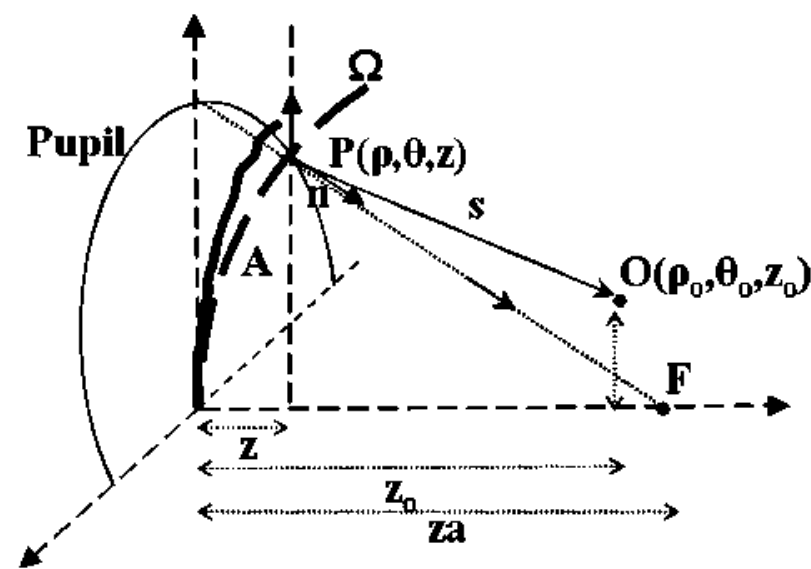

Fig. 1. Geometry showing the coordinates and distances used in the diffraction integral analysis. $P$, point at the ideal spherical wavefront $(\Omega)$; $O$, observation point; $F$, paraxial focal point

$$
u(P)=\frac{1}{4 \pi} \iint_{A}\left\{u \frac{\partial}{\partial n}\left(\frac{e^{i k s}}{s}\right)-\left(\frac{e^{i k s}}{s}\right) \frac{\partial u}{\partial n}\right\} \mathrm{d} A,
$$

where $\partial / 2 n$ denotes differentiation along the inward normal to surface $A, s$ is the distance from point $P$ in surface $A$ to the observation point $O$, and $A$ is the part of the ideal spherical wavefront surface $(\Omega)$ bounded by the exit pupil (see Fig. 1).

For points located at $A$, we defined a local Frenet trihedron reference system (i.e., an orthonormal basis of three vectors: The unit tangent, the unit normal, and the unit binormal) where $n$ is the normal coordinate to $A$. We assumed that the optical field $u$ propagates paraxially in the vicinity of $A$. This assumption is valid because we can define the vicinity of $A$ as small as desired. Hence, the phase of the optical field $u$ for points located at $A$ can be expressed as the addition of two terms: A term containing the wavefront aberration $k W$, and a term considering the phase dependence with the local propagation of the wavefront $k n$. Therefore $u$ is given by the equation

$$
u=e^{i h(W+n)} .
$$

With Eq. (2), we evaluated the partial derivatives in Eq. (1). After some calculations, we obtained

$$
\begin{aligned}
u(P)= & \frac{1}{4 \pi} \iint_{A} e^{i k+W+n)}\left\{\frac{e^{i k s}}{s}\left(i k-\frac{1}{s}\right) \frac{\partial s}{\partial n}\right. \\
& \left.-i k \frac{e^{i k s}}{s}\left(1+\frac{\partial W}{\partial n}\right)\right\} \mathrm{d} A .
\end{aligned}
$$

We used the usual approximation

$$
i k-\frac{1}{s} \simeq i k .
$$

This approximation is justified because the distance $s$ (distance from any point in $\Omega$ to $O$ ) is much larger than the wavelength. Under this approximation, $\mathrm{Eq}$. (3) is reduced to

$$
u(P)=-\frac{i k}{4 \pi} \iint_{A} \frac{e^{i k(W+\eta++s)}}{s}\left\{\left(\frac{\partial W}{\partial n}-\frac{\partial s}{\partial n}\right)+1\right\} \mathrm{d} A .
$$

Equation (5) can be expressed as

$$
u(P)=-\frac{i k}{4 \pi} \iint_{A} \frac{e^{i k(W+n+s)}}{s}(K+1) \mathrm{d} A,
$$

where $K$ can be interpreted as the inclination factor of a Fresnel-Kirchhoff integral kernel type [13]:

$$
K=\frac{\partial W}{\partial n}-\frac{\partial s}{\partial n}
$$

Using cylindrical coordinates, the distance $s=|\overrightarrow{P O}|$ (Fig. 1) is 


$$
s=\sqrt{\rho_{o}^{2}+\rho^{2}+\left(z_{o}-z\right)^{2}-2 \rho_{o} \rho \cos \left(\theta-\theta_{o}\right)},
$$

where the coordinates of $P$ and $O$ are $\{\rho, \theta, z\}$ and $\left\{\rho_{o}, \theta_{o}, z_{o}\right\}$, respectively.

\section{ROTATIONALLY SYMMETRIC DIFFRACTION INTEGRAL}

For some applications, rotational symmetry along the optical axis can be assumed. In this case the diffraction integral is one dimensional, and the spatial coordinates of a point in the space are $\{\rho, z\}$. Functions $s, W$, and $K$ depend only on $\rho$. We can now express $s$ [Eq. (8)] as

$$
s(\rho)=\sqrt{\left(\rho_{0}-\rho\right)^{2}+\left(z_{0}-z\right)^{2}} .
$$

The wave aberration contains only rotationally symmetric terms. For simplicity, we assume that primary spherical aberration is the only contribution.

$$
W(\rho)=W_{4(1)} \rho^{4} .
$$

Applying the chain rule to Eq. (8), we get

$$
K\left(\rho, \rho_{\theta}, z_{0}\right)=\frac{\partial W(\rho)}{\partial n}-\frac{\partial s(\rho)}{\partial n}=\frac{\partial \rho}{\partial n}\left(\frac{\partial W(\rho)}{\partial \rho}-\frac{\partial s(\rho)}{\partial \rho}\right) .
$$

Coordinates $\{\rho, z\}$ of a point in the ideal spherical wavefront are related by the sag equation of a sphere:

$$
z=z a-\sqrt{z a^{2}-\rho^{2}},
$$

where $z a$ is the radius of curvature of the ideal spherical wavefront (i.e., the $z$ coordinate of the paraxial image point). From Fig. 1 we could derive an explicit relation for

$$
\frac{\partial \rho}{\partial n}=\cos (\rho, n)=-\cos (-\rho, n)=\frac{-\rho}{\sqrt{\rho^{2}+(z a-z)^{2}}} .
$$

After applying some calculations, we obtained

$$
\frac{\partial s(\rho)}{\partial \rho}=\frac{\sqrt{z a^{2}-\rho^{2}}\left(\rho-\rho_{o}\right)-\rho\left(z_{o}-z\right)}{\sqrt{\left(z a^{2}-\rho^{2}\right)\left(\rho_{o}-\rho\right)^{2}+\left(z a^{2}-\rho^{2}\right)\left(z_{o}-z\right)^{2}}} .
$$

Using Eqs. (11)-(14), we derived an equation for $K\left(\rho, \rho_{o}, z_{o}\right)$ as a function of $\rho, \rho_{o}$, and $z_{\sigma}$. Finally, using the equation of $K\left(\rho, \rho_{o}, z_{o}\right)$ and $\mathrm{Eq}$. (9), we obtained an explicit relation for the one-dimensional diffraction integral:

$$
u\left(\rho_{0}, z_{0}\right)=-\frac{i k}{4 \pi} \int_{C} \frac{e^{i k(W(\rho)+s(\rho) !}}{s(\rho)}\left(K\left(\rho, \rho_{0}, z_{0}\right)+1\right) \mathrm{d} \rho,
$$

where $C$ is the space curve defining the ideal spherical wavefront in a two-dimensional space. Note that $n(\rho)=0$ for all points $\{\rho, z\}$ located at $C$, as $n$ is the normal coordinate with the origin of the coordinate references located at the space curve $C$.

\section{APPROXIMATIONS TO THE DIFFRACTION INTEGRAL}

Integral (6), and its associated one-dimensional version (15), is the exact diffraction integral following Kirchhoff's theorem. We studied different approximations for this in- tegral in order to evaluate which ones can be justified to compute intensities on the human retina.

1. Constant amplitude approximation. The inclination factor is considered negligible $(K \sim 0)$, and the distance $1 / s$ is replaced with $1 / z_{0}$ in the integration domain. In doing so, the amplitude of the complex integral kernel of Eq. (7) is constant.

2. Fresnel approximation. Term $s$ in the exponential is expanded in the power series, and terms higher than the quadratic term are neglected.

$$
\begin{aligned}
s \sim & \left(z_{o}-z a\right)-\frac{\rho \rho_{o} \cos \left(\theta-\theta_{0}\right)}{\left(z_{o}-z a\right)}+\frac{\rho^{2}}{2\left(z_{o}-z a\right)} \\
& -\frac{\left(\rho \rho_{o} \cos \left(\theta-\theta_{0}\right)\right)^{2}}{2\left(z_{0}-z a\right)^{3}} .
\end{aligned}
$$

3. Fraunhofer approximation. In addition to the Fresnel approximation, the quadratic term of the series expansion of $s$ is also neglected.

$$
s \sim\left(z_{o}-z a\right)-\frac{\rho \rho_{o} \cos \left(\theta-\theta_{o}\right)}{\left(z_{o}-z a\right)} .
$$

\section{NUMERICAL EVALUATION OF DIFFRACTION INTEGRALS}

Integrals (6) and (15) and their approximations are computed numerically. There is extensive work in the literature proposing efficient and accurate algorithms to evaluate diffraction integrals. Standard algorithms divide the integration domain into subdomains and then approximate locally the integrand in each subdomain by expressions that can be integrated analytically. A classical algorithm, proposed by Hopkins and Yzuel , approximates linearly the amplitude and phase. We applied an algorithm proposed by Stamnes and collegeues, the socalled Stamnes-Spjelkavik-Pedersen (SSP) algorithm, where a parabolic approximation is applied to the phase and amplitude. The SSP algorithm was implemented to solve the one-dimensional integral [Eq. (6)]. The twodimensional integral [Eq. (15)] was evaluated by a twostep process : First, the application of the SSP algorithm to the first integration over the angular variable for a number of constant values of the radial coordinate; second, the integration of values from the first integration using a Gauss-Legendre integration formula. The algorithm was implemented in code written in MatLab and $\mathrm{C}$ to optimize the efficiency of the computing time. In order to evaluate the efficiency of the algorithm, we compared it to a generic integral solver available from Matlab (quad function) that makes use of an adaptive Simpson quadrature method.

\section{SIMULATIONS}

The method that we have developed in this study is valid for any type of eye model and wave aberration. The wave aberrations can be computed theoretically or measured experimentally with a wavefront sensor such as laser ray 
tracing or a Hartmann-Shack wavefront sensor. For illustration purposes, as an example, we used a pseudoaphakic eye model with some amount of simulated wave aberrations.

In pseudoaphakic eyes the crystalline lens is replaced with an intraocular lens. In such a case, it is usually necessary to compute the intensity reaching the retina when the eye is looking at different distances. We used a pseudoaphakic eye model (see Subsection 2.1 in Barbero and Marcos ), modeling the cornea and intraocular lens as a set of concentric surfaces and separated by homogeneous media. We set the distance from the exit pupil to the retina $\left(z_{0}=20.98 \mathrm{~mm}\right)$ equal to the focal length of the eye model. We computed the intensities on the retina for a point object located on axis at different distances.

We performed two different analyses. (1) An axial intensity evaluation where we computed intensities at the image point defined by the intersection of the optical axis with the retina for different object point vergences [object location in diopters $(\Delta)$ ] ranging from $-4 \mathrm{D}$ to $4 \mathrm{D}$ in steps of $0.2 \mathrm{D}$. (2) A transverse intensity evaluation where we computed intensities at points in a transverse plane located at the focal plane or at an out-of-focus plane.

We modeled a $6.5 \mathrm{~mm}$ diameter pupil (in order to test a limit case of large pupil size), and we used different aberration levels.

In order to validate the efficiency of the numerical implemented algorithm (SSP code), we compared it to the Matlab quad algorithm. We tested the validity of the usually applied constant amplitude approximation, assuming for simplicity a rotationally symmetric wavefront. The conclusions about the validity of the SSP algorithm and the constant amplitude approximation would be the same in the nonsymmetrical case because the approximation mainly affects the $z$ coordinate. We simulated a wavefront aberration containing only the spherical Zernike term $Z_{40}=1 \mu \mathrm{m}$.

The SSP algorithm accuracy and efficiency depends on the number of subdomains in which the pupil is divided and on the shape of the wave aberration function. Following Stamnes, we evaluated the relative accuracy of the SSP algorithm as a function of the number of subdomains using the percentage error. The percentage error was evaluated as the mean percentage error of the intensity computation at all points in an axial or transverse intensity analysis, where the reference values were obtained using a large number of subdomains. We evaluated the algorithm efficiency by estimating the computation time needed for the intensity evaluation of one point. We used an AMD Athlon(tm) $64 \times 2$ dual core processor with 3 MBytes of RAM memory. We used two different types of wave aberrations: (1) a rotationally symmetric wave aberration $\left(Z_{40}=1 \mu \mathrm{m}\right)$ and (2) an astigmatic wave aberration $\left(Z_{22}=1 \mu \mathrm{m}\right)$.

Finally, we performed an axial and transverse intensity analysis to evaluate the accuracy of the Fresnel and Fraunhofer approximations. For this test we used an astigmatic wavefront $\left(Z_{22}=1 \mu \mathrm{m}\right.$ ). In addition, we also computed intensities by applying the conventional procedure

. In this procedure the wavefront aberration is considered with respect to the exit pupil plane, and the Fraunhofer diffraction integral is used. In the axial intensity analysis, the defocus is introduced as an ad hoc addition of a focus term $\left(Z_{20}\right)$ to the phase.

\section{RESULTS}

Figure 2 shows the axial and transverse intensity computations using the SSP and quad Matlab algorithms. Whereas the results were very similar when computing the axial intensities [Fig. 2(a)], we found significant differences in the transverse intensities [Fig. 2(b)], with some artifacts appearing when using the Simpson algorithm in the intensity computation for distances larger than $0.4 \mathrm{~mm}$ off axis. Furthermore, the time employed for a single observation point to compute the exact integral using the Simpson versus the SSP algorithm was larger: $3.95 \mathrm{~s}$ versus $0.99 \mathrm{~s}$.

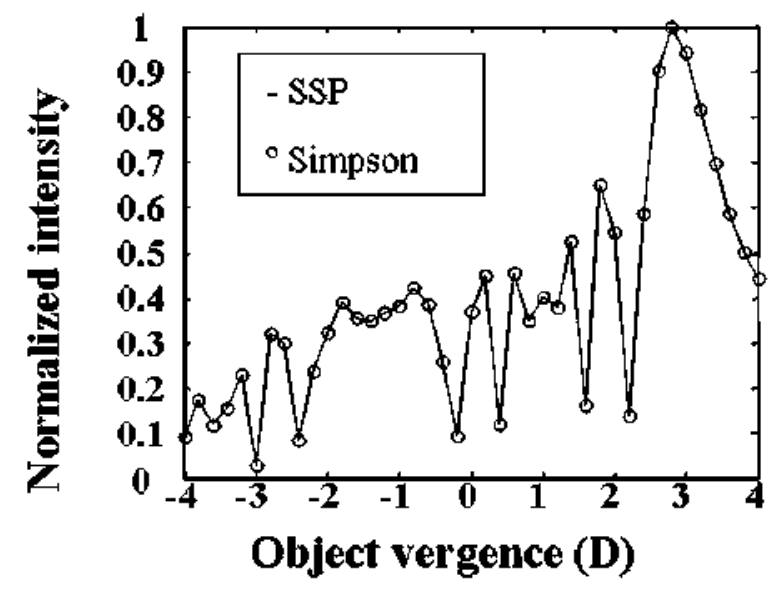

(a)

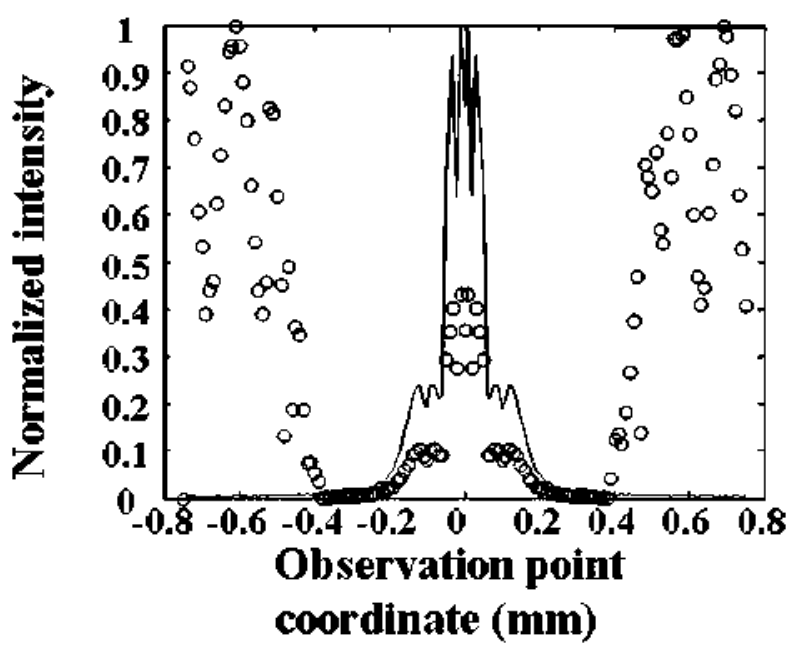

(b)

Fig. 2. Normalized intensity distribution using a pseudoaphakic eye model : (a) along the optical axis for different object vergences and (b) along the plane perpendicular to the optical axis at an image plane located $1 \mathrm{~mm}$ out of the paraxial fociss. The intensities computed using the SSP algorithm and the Simpson method are represented by a solid anve and black circles, respectively. The pupil radius was set to $3.25 \mathrm{~mm}$, the Zernike spherical aberration $\left(Z_{11}\right)$ was set to $1 \mu \mathrm{m}$, and the wavelength was set to $0.555 \mu \mathrm{m}$ (photopic peak). 
Table 1. Percentage Errors and Computation Times (for One Observation Point) of the SSP

Algorithm as a Function of the Number of

Subdomains in the Radial $\left(N_{r}\right)$ and Angular Coordinates $\left(N_{\theta}\right)$

\begin{tabular}{cccc}
\hline$N_{\theta} / N_{r}$ & 59 & 78 & 90 \\
\hline On axis intensity analysis: $Z_{40}=1 \mu \mathrm{m}$ & \\
1 & $63.55 \%(0.04 \mathrm{~s})$ & $2.73 \%(0.06 \mathrm{~s})$ & $0.002 \%(0.07 \mathrm{~s})$ \\
On axis intensity analysis: $Z_{22}=1 \mu \mathrm{m}$ & \\
60 & $59.12 \%(0.32 \mathrm{~s})$ & $4.67 \%(0.67 \mathrm{~s})$ & $4.59 \%(1.01 \mathrm{~s})$ \\
200 & $17.77 \%(0.24 \mathrm{~s})$ & $0.45 \%(0.89 \mathrm{~s})$ & $0.2 \%(1.44 \mathrm{~s})$ \\
300 & $12.91 \%(0.27 \mathrm{~s})$ & $0.31 \%(1.03 \mathrm{~s})$ & $0.2 \%(1.67 \mathrm{~s})$ \\
\hline$N_{\theta} / N_{r}$ & 200 & 400 & 600 \\
\hline Transverse intensity analysis: $Z_{40}=1 \mu \mathrm{m}$ & \\
59 & $76.19 \%(0.76 \mathrm{~s})$ & $72.04 \%(1.48 \mathrm{~s})$ & $71 \%(2.2 \mathrm{~s})$ \\
146 & $1.92 \%(1.9 \mathrm{~s})$ & $0.89 \%(3.7 \mathrm{~s})$ & $0.35 \%(5.53 \mathrm{~s})$ \\
5860 & $1.69 \%(76.16 \mathrm{~s})$ & $0.88 \%(149.1 \mathrm{~s})$ & $0.34 \%(220.97 \mathrm{~s})$ \\
\hline$N_{\theta} / N_{r}$ & 400 & 600 & 1200 \\
\hline Transverse intensity analysis: $Z_{22}=1 \mu \mathrm{m}$ & \\
98 & $83.83 \%(2.96 \mathrm{~s})$ & $80.87 \%(4.42 \mathrm{~s})$ & $78.01 \%(8.75 \mathrm{~s})$ \\
117 & $5.53 \%(3.54 \mathrm{~s})$ & $4.04 \%(5.31 \mathrm{~s})$ & $0.49 \%(10.51 \mathrm{~s})$ \\
5860 & $4.68 \%(177.19 \mathrm{~s})$ & $3.08 \%(264.9 \mathrm{~s})$ & $0.49 \%(524.32 \mathrm{~s})$ \\
\hline
\end{tabular}

Table 1 shows the percentage errors using different numbers of subdomains and two different wave aberrations. We found that the number of subdomains, and hence the computation demands, needed to achieve an accurate result-less than $0.5 \%$ of the percentage error, following Stamnes' criteria-are much higher for the transverse intensity analysis and the asymmetric wavefront than for the axial intensity analysis and the symmetric wavefront. For the symmetric wavefront and the simplest case (the axial analysis), a radial sampling of 90 points was sufficient. When performing a transverse in-

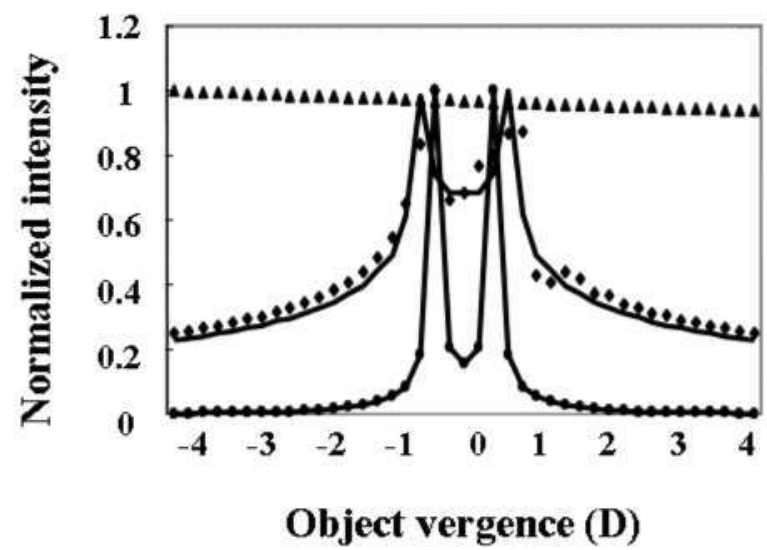

Fig. 3. Normalized intensity distribution along the optical axis for different object vergences using a pseudoaphakic eye model applying the constant amplitude approximation (solid curve), Fresnel approximation (circles), Fraunhofer approximation (triangles), and the conventional procedure (solid curve with circles) as explained in Section 6 . The intensity was computed using the SSP algorithm. The pupil radius was set to $3.25 \mathrm{~mm}$, the Zernike astigmatic aberration $\left(Z_{22}\right)$ was set to $1 \mu \mathrm{m}$, and the wavelength was set to $0.555 \mu \mathrm{m}$. tensity analysis, at least 600 angular and 146 radial subdomains were necessary. The sampling demands were higher for the asymmetric wavefront, whereas for the transverse analysis at least 1200 angular subdomains were necessary.

Figure 3 shows the normalized intensity distribution for the axial intensity analysis (asymmetric wavefront) applying the constant amplitude approximation, the Fresnel and Fraunhofer approximations, and the conventional procedure explained before. The Fraunhofer approximation did not provide a reasonable estimation at all, whereas the Fresnel approximation could be justified but still presented some artifacts in the computations. The conventional procedure showed important differences in the intensity computations with respect to our computations, especially regarding the decay of intensity out of focus.

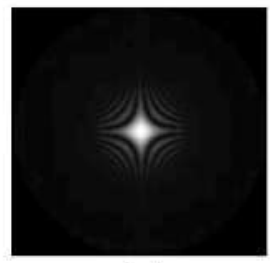

(a)

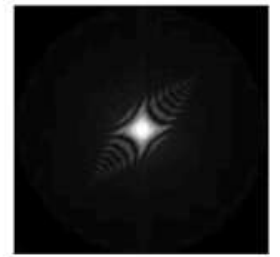

(c)

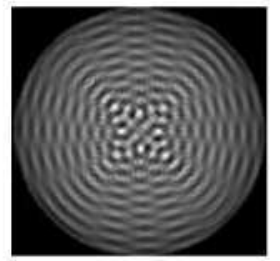

(e)

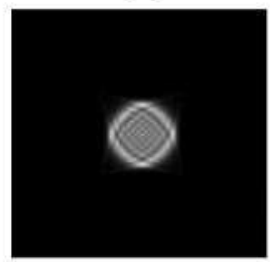

(g)

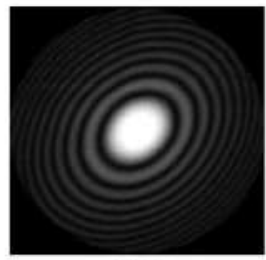

(b)

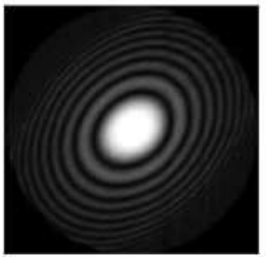

(d)

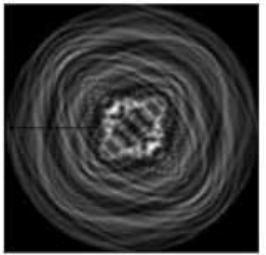

(f)

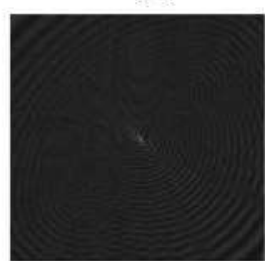

(h)
Fig. 4. Intensity distribution at points located in a transverse plane in focus and 3 diopters (D) out of focus. The dimensions are a square of $0.1 \mathrm{~mm}$. Shown are the constant amplitude approximation at focus (a) and out of focus (b); the Fresnel approximation at focus (c) and out of focus (d); and the. Fraunhofer approximation at focus (e) and out of focus (f). The intensity was computed using the two-dimensional SSP algorithm. The conventional procedure, using a FFT algorithm, was used to generate the intensity images at focus (g) and out of focus (h). The pupil radius was set to $3.25 \mathrm{~mm}$, the Zernike astigmatic aberration $\left(Z_{22}\right)$ was set to $1 \mu \mathrm{m}$, and the wavelength is set to $0.555 \mu \mathrm{m}$. 
The same computations were performed in a transverse analysis, and the results are shown in Fig. 4. For this case, the Fraunhofer approximation failed again, but in addition, the Fresnel approximation presented some artifacts that contrasted with the constant amplitude approximation at the plane of best focus. The intensity distribution both by scale and internal structure is also significantly different in the case of the conventional procedure.

\section{DISCUSSION}

Using Kirchhoff's diffraction theory, we have obtained the diffraction integral necessary to compute the optical field on the retina, knowing the wave aberration with respect to the ideal spherical wavefront. To derive that integral, besides applying the usual procedure in Kirchhoff's theory (including Kirchhoff's boundary condition and the approximation $i k \gg 1 / s$ ), we included an extra step. We assumed that the optical field $u$ propagates paraxially in the vicinity of the ideal spherical wavefront $A$. As $\partial u / \partial n$ is evaluated exclusively on surface $A$, the paraxial condition is sufficient to be valid in a region as close as needed to $A$. Therefore, except in the case of singularities in the optical field, this approximation is acceptable.

Two algorithms were compared to evaluate the derived diffraction integral: A generic adaptive Simpson quadrature method and an efficient algorithm based on a local quadratic approximation to the amplitude and phase (SSP algorithm). The Simpson method presented some inconsistencies when computing intensities off axis. In addition, the SSP algorithm is more efficient in terms of computing time.

We point out that, although the methodology is valid for any type of wave aberration, these results and their conclusions are strictly justified only for the pupil size and aberration levels described in Section 6 .

The results of Figs. 3 and 4 showed that the Fresnel approximation can be justified to compute intensities on the retina, although some artifacts could appear in some cases (Fig. 4). As the computing time (SSP algorithm) using the constant amplitude approximation is similar to that using the Fresnel approximation (0.01\% difference), we recommend using the constant amplitude approximation; i.e., the inclination factor is zero, and $1 / s$ is replaced with $1 / z_{0}$. This approximation reduced the complexity of the exact integral and saved computation time.

The Fraunhofer approximation results were quite different from the exact results even at the focal plane. This occurred because the phase in the Fraunhofer approximation in the ideal spherical wavefront differs from a constant, as it is nearly the case in the other approximations.

Finally, an important result of this work shows that the conventional procedure used in the visual optics community to compute intensities at the retinal plane (PSFs) differs significantly from the exact results. In the conventional procedure, the fact that the wave aberration is assumed to be considered with respect to the exit pupil generates phase changes several times larger than the wavelength over the pupil size, therefore producing fast phase shifts. This explains why typical PSF images show strong oscillations in the intensity pattern.

\section{ACKNOWLEDGMENTS}

We thank Jacob Rubinstein for his valuable comments. We also thank Rhiannon Borgia for her corrections to the manuscript. The authors acknowledge funding from an EURYI Award (ESF-EUROHORCs), from FIS2005-04382 (Ministerio de Educacin y Ciencia) to $\mathrm{S}$. Marcos, and from the I3P (CSIC) Program to S. Barbero.

\section{REFERENCES}

B. Vohnsen, "Photoreceptor waveguides and effective retinal image quality," J. Opt. Soc. Am. A 24, 597-607 (2007).

J. J. Stamnes, Waves in Focal Regions: Propagation, Diffraction and Focusing of Light, Sound and Water Waves (IOP, 1986).

J. Perez, D. Mas, C. Illueca, J. J. Miret, C. Vazquez, and C. Hernandez, "Complete algorithm for the calculation of light patterns inside the ocular media," J. Opt. Soc. Am. A 52, 1161-1176 (2005).

D. A. Atchison and G. Smith, Optics of the Human Eye (Butterworth-Heinemann, 2000).

S. Chavez-Cerda, "Nonparaxial effects in visual imaging of the Emsley schematic eye," presented at the Third European Meeting in Physiological Optics, City University, London, September 7-9, 2006.

R. Navarro and E. Moreno-Barriuso, "Laser ray-tracing method for optical testing," Opt. Lett. 24, 951-953 (1999).

E. Moreno-Barriuso and R. Navarro, "Laser ray tracing versus Hartmann-Shack sensor for measuring optical aberrations in the human eye," J. Opt. Soc. Am. A 17, 974-985 (2000).

A. Castro, S. Barbero, and S. Marcos, "A reconstmiction technique to estimate the gradient-index distribution of the crystalline lens lising ray aberration data in wino," presented at the 2007 ARVO Anmual Meting, Fort Landerdale, Florida, May 6-10, 2007.

H. G. Kraus, "Huygens-Fresnel-Kirchhoff wavefront diffraction formulation: Spherical waves," J. Opt. Soc. Am A 6, 1196-1205 (1989).

P. Artal, "Calculations of two-dimensional foveal retinal images in real eyes," J. Opt. Soc. Am. A 7, 1374-1380 $(1990)$.

S. Marcos and S. A. Burns, "On the symmetry between eyes of wavefront aberration and cone directionality" Vision Res. 40, 2437-2447 (2000).

G. Kirchhoff, "Zur Theorie der Lichtstrahlen," Ann. Phys. 254, 663-695 (1883).

M. Born and E. Wolf, Principles of Optics: Electromagnetic Theory of Propagation, Interference and Diffraction of Light (Pergamon, 1980).

E. Wolf and E. W. Marchand, "Comparison of Kirchhoff and the Rayleigh-Sommerfeld theories of diffraction at an aperture," J. Opt. Soc. Am. 54, 587-594 (1964).

H. H. Hopkins and M. J. Yzuel, "The computation of diffraction patterms in the presence of aberrations," Opt. Acta 17, 157-182(1970).

J. J. Stamnes, B. Spjelkavik, and H. M. Pedersen, "Evaluation of diffraction integrals using local and phase amplitude approximations," Opt. Acta 30, 207-222 (1983). $\mathrm{S}$. Barbero and S. Marcos, "Analytical tools for customized design of monofocal intraocular lenses," Opt. Express 15, 8576-8591 (2007).

X. Cheng, A. Bradley, and L. N. Thibos, "Predicting subjective judgment of best focus with objective image quality metriss," Aust. Math. Soc. Gaz. 4, 310-321 (2004). S. Marcos, S. Barbero, and I. Jimenez-Alfaro, "Optical quality and depth-of-field of eyes implanted with spherical and aspheric intraocular lenses," J. Refract. Surg. 21, 223-235 (2005). 